\title{
« Vers carminiformes » et « oraison soluë » dans les œuvres de Rabelais : poésie et poétique de la constellation
}

\author{
Julien LEBRETON \\ Université Paris-Sorbonne \\ École doctorale V « Concepts et Langages » \\ lebreton.julien1@gmail.com
}

Recibido: $21 / 08 / 2012$

Aceptado: 23/01/2013

\begin{abstract}
Résumé
L'article a pour objet l'étude de la poésie dans l'œuvre de Rabelais. L'analyse est menée à partir de la langue poétique de Rabelais qui touche avant tout le lecteur par sa matérialité et par sa musicalité. Toute l'œuvre de Rabelais peut se caractériser comme poétique en conférant à cet adjectif toute l'étendue de contradictions dont il est susceptible. Ainsi, la poésie, à travers les «romans » rabelaisiens sort grandie, ou plutôt elle ressort agrandie, car elle est capable d'accueillir en son sein les alliances de tous les genres. Nous sommes ainsi conduit à nous demander quelle est la fonction de la poésie chez Rabelais : se révèle-t-elle être un instrument de diversification ? Si tel est le cas, il s'agit de statuer sur la définition et la délimitation de la poésie de Rabelais qui constitue une composante primordiale de l'œuvre rabelaisienne. Loin d'être une entrave à la construction du sens, elle serait au contraire un atout.
\end{abstract}

Mots clés : poésie, prose, hybridité, parodie.

\section{« Vers carminiformes » y « oraison soluë » en las obras de Rabelais: poesía y poética de la constelación}

\section{Resumen}

El artículo se centra en el estudio de la poesía en la obra de Rabelais. El análisis se lleva a cabo a partir del lenguaje poético del autor, que emociona al lector principalmente por su materialidad y su musicalidad. Toda la obra de Rabelais puede calificarse como poética, confiriéndole a este adjetivo todas las contradicciones de las que es susceptible. De ese modo, la poesía, a través de las «novelas» rabelaisianas se enriquece, o más bien sale enriquecida, ya que es capaz de albergar en su interior la unión de todos los géneros. Llegamos así a preguntarnos cuál es la función de la poesía en la obra de Rabelais y si ésta se revela como un instrumento de diversificación. Si ese es el caso, habrá que tomar una decisión sobre la definición y la delimitación de la poesía de Rabelais, que constituye un componente esencial de la obra rabelaisiana y que, lejos de ser un obstáculo para la construcción del sentido, sería al contrario, una ventaja.

Palabras clave: poesía, prosa, hibridismo, parodia. 


\title{
«Vers carminiformes» and « oraison soluë » in Rabelais’s work: poetry and poetics of constellation
}

\begin{abstract}
The article focuses on the study of poetry in the works of Rabelais. I will analyze Rabelais's poetic language, which moves the reader mainly through its materiality and musicality. Rabelais's work may be characterized as poetic inasmuch as this adjective is endowed with the full range of contradictions that it is liable to have. Thus, poetry, in the Rabelaisian "novels", emerges richer - or rather, enriched-, because it is capable of encompassing alliances of all genres. This leads us to wonder what the function of poetry is in Rabelais's works: is it an instrument of diversification? If this is the case, it is necessary to define the scope of Rabelais's poetry, which constitutes an essential component of his oeuvre. Far from being an obstacle to the construction of meaning, his poetry would be, on the contrary, an asset.
\end{abstract}

Keywords: poetry, prose, hybridity, parody.

\section{Referencia normalizada}

Lebreton, J. (2013). “"Vers carminiformes » et « oraison soluë» dans les œuvres de Rabelais : poésie et poétique de la constellation”. Thélème, Vol. 28, 171-186.

Alors qu'il demeure essentiellement perçu comme un prosateur, il nous paraît opportun de réhabiliter Rabelais en tant que poète précurseur (Haskovec, 1908 : 5660 ; Plattard, 1911 : 90-108; Plattard, 1912 : 291-304). C'est plutôt cette image qu'il renvoie à ses contemporains, d'autant plus qu'il s'inscrit dans une mouvance littéraire bien singulière, celle de la Grande Rhétorique ${ }^{1}$. Il faut également préciser que Rabelais a entretenu des liens très étroits avec la Pléiade ${ }^{2}$. Parmi les auteurs les

\footnotetext{
${ }^{1}$ Voir Lebègue R. (1958) : «Rabelais et les grands rhétoriqueurs », Lettres romanes, 12, 5-18. Pour sa part, Lebègue a analysé certaines parentés d'idées et de style avec notamment Guillaume Cretin, Jean Lemaire et Jean Molinet. En revanche, Rigolot F. (1978) : dans « Rabelais rhétoriqueur », Cahiers de l'Association Internationale des Etudes Françaises, XXX, 87-103, repris dans Le Texte de la Renaissance. Des Rhétoriqueurs à Montaigne (1982) : Genève, Droz, 107-121, exprime ses réserves envers le collationnement des sources et les rapprochements biographiques et stylistiques effectués par ses devanciers. Il suggère de "saisir le rhétoriqueur en Rabelais pour ainsi dire à son corps défendant, afin de rendre compte de sa pratique d'écrivain plutôt que des composantes historiquement marquées de son texte»; De son côté, Chambon J.-P. (1993) : dans "Andrieu de la Vigne, source de Rabelais ? ", Revue des études rabelaisiennes, XXIX, 57-61, rapproche Rabelais du Grand Rhétoriqueur Andrieu de la Vigne. Il se demande si ce dernier n'a pas inspiré le style du Cri apotropaïque de l'entrée de Thélème, dont le matériel lexical et rimique offre pour ainsi dire des analogies avec le Roy de la Bazoche; Voir également De Cornulier B. (2000): "Rabelais Grand Rhétoriqueur. L'enchaînement dans l'Inscription mise sur la grande porte de Thélème », Études Rabelaisiennes, XXXIX, 111-124.

${ }^{2}$ Voir Fontaine M.-M. (2001) : « Le système des références dans le Cinquiesme Livre et les 'éruditionnés' de Rabelais », dans Le Cinquiesme Livre, Actes du colloque international de Rome réunis et
} 
plus imités et les plus suggérés, figurent en tête de liste Mellin de Saint-Gelais et Clément Marot. Mais, au fil des « romans » rabelaisiens, d'autres poètes sont également mentionnés : de Guillaume Crétin (Slerca, 1996: 586-589) ou encore le poète interlope, François de Montcorbier (Thuasne, 1911) sans oublier Francesco Colonna ${ }^{3}$. Rabelais se plaît à imiter des modèles célèbres, dès lors, l'imitatio comporte un aspect essentiel, perceptible à travers le traitement qu'il fait subir aux poèmes qu'il imite ou emprunte aux Rhétoriqueurs. Pourtant, chez Rabelais, il n'est pas toujours aisé de déceler dans l'usage du vers la part du clin d'œil intertextuel, celle de la fantaisie verbale, celle de la solennité accusée, et finalement celle du détournement parodique. Dans l'œuvre rabelaisienne, mais surtout dans le Pantagruel et le Gargantua, tous les modèles dont se réclame Rabelais sont comiques ou subissent un traitement parodique. Dès lors :

Pantagruel et Gargantua sont deux textes construits à partir d'un modèle inscrit dans le champ d'une sub-littérature absurdement comique où les scénarios du roman chevaleresque traditionnel s'entremêlent à des histoires légendaires et fantastiques (Pilar Suárez, 2006 : 202).

Qu'est-ce que la poésie au XVI ${ }^{\mathrm{e}}$ siècle? Trouver des critères pour définir le genre n'est guère évident. À la Renaissance, le discours théorique sur la poésie se

publiés par Giacone F, Genève, Droz, 501-552.Elle formule la thèse selon laquelle une véritable complicité s'est tissée entre Rabelais et la Pléiade. Par ailleurs, Pot O. (1988) : dans «Ronsard et Panurge à Ganabin », Études Rabelaisiennes, XXII, 7-26, propose de lire dans le personnage de Rodilardus, un avatar parodique de Ronsard dans le sillage de Berrong R. M. (1980, "The Cinquième Livre and the 'querelle de la Deffence' », Études Rabelaisiennes, XV, 145-159), lequel soulignait la tension d'ordre esthétique entre les deux écrivains. Pour HuchonM. (1986) : dans «Rabelais et les “'genres d'escrire', dans Rabelais's Incomparable Book, ed. La Charité R. C., Lexington, French Forum Publishers, note 32, 245, il faut relier ces passages «à des recherches antérieures occultées par la fortune de la Deffence $»$.

${ }^{3}$ Nous pouvons songer, en tout particulier au Pantagruel, chap. XXVII, 308-311 dans Euvres complètes, éd. de Huchon M. avec la collaboration de Moreau F. (1994):Paris, Gallimard, «Bibliothèque de la Pléiade». Désormais toutes les références aux textes rabelaisiens renverront à cette édition. Dans ce chapitre, lors du répit qui leur est accordé, avant la bataille finale contre les Dipsodes, Pantagruel et ses compagnons célèbrent leur première victoire, remportée sur "six cens soixante morpions». Ce moment s'avère ainsi propice à l'érection d'un autel à la mémoire des héros victorieux. Le texte indique explicitement, dans le titre du chapitre par exemple «Comment Pantagruel droissa un Trophée en mémoire de leur prouesse », l'appartenance de ce poème au genre du " trophée ». Du reste, précisons que l'on trouve, dans Le Songe de Poliphile (Hypnerotomachia Poliphili), éd. Polizzi G. (1994) : Paris, Imprimerie Nationale, Paris, Livre premier, chap. XIX, un «trophée » en forme de médaillon accompagné de sa description. Gravé sur le socle d'un obélisque à côté de compositions hiéroglyphiques, cet ensemble véhicule une profondeur allégorique dont le trophée de Pantagruel semble être dépourvu. Il faut également souligner que les poèmes figurés connaissent au XVI siècle et au XVII ${ }^{\mathrm{e}}$ siècle un succès inégalé, probablement parce que l'Anthologie grecque a été publiée en 1494, réimprimée à plusieurs reprises, notamment à Paris en 1531, et que des carmina figurata chrétiens circulaient grâce à l'édition de 1503 des œuvres de Raban Maur, un poète du $\mathrm{IX}^{\mathrm{e}}$ siècle et disciple d'Alcuin, auteur d'un De laudibus sanctae crucis. 
confond avec la poétique, c'est ainsi que l'on pourrait considérer toute l'œuvre de Rabelais comme poétique en conférant à cet adjectif toute l'étendue de contradictions dont il est porteur. Le flou conceptuel autour de la poésie repose sur la diversité des modèles : s'agit-il d'un « savoir spécialisé voisin de la rhétorique » (Lecointe, 2001 : 63), correspondant à un savoir technique, celui des règles prosodiques, ou du « lieu de récapitulation de la totalité du savoir, dans une implicite concurrence avec la théologie-métaphysique » (Lecointe, 2001 : 63), correspondant à une vision très noble de la poésie comme « fureur»?

Dans les « romans » de Rabelais, où apparaissent les formes poétiques ? La situation est-elle la même dans chaque « roman »? D'autre part, s'agit-il de formes fixes ou de formes libres ? Quel mètre Rabelais utilise-t-il de préférence ? Sachant que le vers le plus utilisé jusqu'à la Pléiade n'est pas encore l'alexandrin, mais le décasyllabe. Comment les formes poétiques s'insèrent-elles dans la trame romanesque ? Comment retentissent-elles par rapport à la prose alentour? Créent-elles un effet de surprise ? Une mise en relief? Un effet ironique ? Apparaissent-elles comme une recherche inutile, ou au contraire revêtent-elles un aspect hiératique en accord avec le contexte ? Prose et poésie se mêlent malicieusement et «à plaisir» dans une «stratégie du mixte » (Molino, 1993 : 3-28 ; Rothstein, 1996 : 35-47 ; Cornilliat, 2002 : 335-356), pour reprendre une expression de Jean Molino. Rabelais est pour ainsi dire, si peu un poète comme les autres que l'on ne pense pas toujours à lui comme à un poète. Il faut rappeler l'hétérogénéité particulièrement frappante de l'œuvre de Rabelais. Dans ses « romans », la notion de mélange, ellemême dépassée par celle de parodie, permet d'aboutir à l'idée du dialogue à la fois intertextuel et formel, entre prose et poésie. L'effet de ce mélange est didactique chez Rabelais: dès lors, l'expression poétique chez Rabelais ne correspondrait-elle pas à la recherche de formes brèves et frappantes, donnant forme à la parodie du discours sentencieux, donc cherchant, à opposer règles et liberté créatrice, la subversion dans les vers venant en dialogue avec la libération de la prose ?

La poésie englobe et dépasse le nécessaire examen des vers inclus dans la prose des « romans » rabelaisiens. C'est ainsi qu'il nous faudrait considérer toutes les facettes et les métamorphoses de ce que la Renaissance pouvait entendre par « poésie » et « art poétique », et dès lors, nous pourrions caractériser l'œuvre de Rabelais dans son ensemble non seulement comme un témoin, mais comme un laboratoire de cette évolution. Voici quelques poèmes qui sont disséminés dans les « romans » rabelaisiens :

\section{Gargantua}

- Le poème liminaire est un dizain adressé aux lecteurs

- «Les Fanfreluches antidotées » trouvées en un monument antique (chap. II : 11-14)

- Épigramme et rondeau du torche-cul (chap. XIII : 39-41)

- Les jeux de Gargantua (chap. XXII : 58-64)

- Inscription mise sus la grande porte de Theleme (chap. LIIII : 141-144) 
- Enigme en prophetie (chap. LVIII : 150-153)

- La harangue de Janotus de Bragmardo (chap. XIX : 51-53)

\section{Pantagruel}

- Le dizain adressé par Hugues Salel à Rabelais (211)

- Épitaphe à Badebec (chap. III : 226)

- Le baragouin latinisant de l'Ecolier Limousin (chap. VI : 232-235)

- Le procès de Baisecul et Humevesne (chap. X-XIII : 250-262).

- Rondeau de Panurge à la dame Parisianne (chap. XXII : 296)

- Les trophées ou dicton victorial (chap XXVII : 309-310)

- Le quatrain écrit au-dessus de la treille la devise d'Epictete (chap. XXX : 325)

Tiers Livre

- Le dizain liminaire à la Reine de Navarre

- Deux distiques prononcés par le narrateur (chap. I et II : 355 et 358)

- Le quatrain en octosyllabes est donné comme provenant de la marraine du grand-père de Panurge (chap. VI : 371)

- Â propos de la «magnifique braguette » de Panurge (chap. VII : 371-373)

- Le huitain (dans lequel est illustré l'argument de Panurge, à savoir que la braguette « est piece premiere de harnois militaire »), qui clôt le chap. VIII (376)

- Les « sors Homeriques et Virgilianes » (chap. X- XII : 379-388)

- Le poème de la Sibylle de Panzoust (chap. XVII : 404)

- Le rondeau de Raminagrobis (chap. XXI : 417)

- Blason du couillon (chap. XXVI : 432-434)

- L'épigramme de Martial (chap. XXVI : 435)

- Blason (chap. XXVIII : 439-441)

- Dialogue entre Trouillogan et Panurge (chap. XXXVI : 463-466)

- Blason du Fou (chap. XXXVIII : 470-473)

- Le plaidoyer du légiste Bridoye (chap. XXXIX-XLIII : 474-487)

- Un poème en Lanternois (chap. XLVII : 495-496)

- Poème (huitain en décasyllabe) qui clôt le Tiers Livre., éloge de la France et le Pantagruélion (chap. LII : 512-513)

\section{Quart Livre}

- Le dizain prononcé par Priape, Prologue (530)

- L'anatomie de Quaresmeprenant (chap. XXX-XXXII : 608-615)

- Le « dizain jolliet» de Panurge (chap. XLIIII : 640)

- Le quatrain de François Villon (chap. LXVII : 700) 


\section{Cinquiesme Livre}

- Le «poème figuré », ou plutôt l'épilénie : un chant accompagnant les vendanges (chap. XLIIII : 831-832)

- Le péan de Panurge (chap. XLV : 834-835)

- Le chant en forme d'autel de Pantagruel, dont la structure n'est pas sans rappeler celle des trophées («Croyez que c'est la fureur poëtique / Du bon Bacchus : ce bon vin eclyptique... » (836), ainsi que le dialogue en vers entre Panurge et Frère Jean mêlant à la fois, dualité de la parole poétique et furor poeticus (chap. XLVI : 835-838)

Quel est le statut de la poésie dans l'œuvre de Rabelais ? À quoi lui est-elle utile ? Et, dans une autre mesure, la poésie chez Rabelais est-elle vouée à être dite, ou à être lue? Au demeurant, jusqu'où peut-elle se dire et/ou se lire? Quelles sont les relations qu'entretiennent prose et poésie dans l'œuvre de Rabelais? C'était d'ailleurs, on le sait, une pratique courante chez les Rhétoriqueurs que de mêler des vers au tissu de la prose. Chez Rabelais, comment se déploie cette écriture poétique capable d'élaborer un monde autre, une langue poétique irréellement fabuleuse et inouïe ? Comme l'écrit Michel Jeanneret :

La langue se prête aussi à toute espèce d'expériences normalement bannies du texte littéraire : fabrication de néologismes, figures étymologiques, calembours et autres effets de paronymie, insertion de termes grecs..., tout l'espace de création que le mot ouvre à l'écrivain semble avoir été parcouru. [...] C'est le grand brassage des genres : la prose, ici où là, est mêlée de vers ; des dissertations érudites, des réminiscences poétiques voisinent avec des contes de bonne femme et de creux bavardages (Jeanneret, $1987:$ 145-147).

Le projet de « Rabelais, rhétoriqueur mutant» (Cornilliat, 1994 : 608), est semblable au grand projet de ses contemporains, celui d'une « œuvre totale », où non seulement la prose serait « rythmisée », mais aussi où tout le langage se trouverait « poétisé ». Du reste, la puissance poétique des « romans » rabelaisiens est particulièrement coruscante. Rabelais « vrai poète » (Guilbaud, 1989: 75) n'a pas à rougir auprès de Rabelais-prosateur. En outre, toute l'œuvre de Rabelais pourrait être considérée comme poétique en conférant à cet adjectif toute l'étendue de contradictions dont il est susceptible. Que l'on songe au chapitre V (Rabelais, Gargantua, 1994 : 17-20), dans lequel la multitude des voix ne fait que résonner et fait vivre le silence du texte. En parfaite analogie avec le chapitre II, il propose une opposition aux «fanfreluches antidotées» par le lien qu'il établit avec les livres et le vin. « Natura abhoret vacuum» l'adage déploie le renversement des effets des premiers mots. Si la nature a horreur du vide, la page est envahie sous un amoncellement de voix ivres vociférant dans tous les replis de la page.

Pensons également au morceau de bravoure que constitue la harangue de Janotus de Bragmardo qui abonde en néologismes comiques et barbarismes hybrides du latin et du français : «O monsieur domine, clochidonnaminornobis. Dea est bonumurbis. Tout le monde s'en sert. Si vostre jument s'en trouve bien : aussi faictnostre 
faculté, que comparata est iumentisinsipientibus : et similis facta est eis, psalmonescio que, si l'avoys je bien quotté en mon paperat, et est unum bonum Achilles, Hen, hen, ehen, hasch.», dit-il pour récupérer à Gargantua les cloches de Notre Dame (Rabelais, Gargantua, 1994 : 52). Chez Rabelais, le mépris de ce langage hermétique des rhétoriqueurs et des légistes, un langage qui martyrise le français et le latin et qui se plaît de ses complexités et de son obscurité, se manifeste à plusieurs reprises dans le cadre de la parodie carnavalesque. Ainsi, lors de l'affaire de Baisecul et d'Humevesne, il endosse le rôle de la mère sotte, prononçant une sentence aussi inintelligible et absconse que la cause même (Rabelais, Pantagruel, $1994: 261)$.

Il reste donc à faire le point sur ce domaine qu'est la poésie, domaine encore peu exploré. Il n'a jusqu'ici été abordé par la critique que de manière partielle ou indirecte $^{4}$. En réalité, on n'a pas encore fait le tour de toutes les facettes poétiques qui se

\footnotetext{
${ }^{4}$ Sans prétendre à l'exhaustivité, nous pensons plus particulièrement aux études suivantes : Stapfer P., Rabelais, sa personne, son génie, son cuvre, Paris, Armand Colin, 1889, consacre un chapitre (chapitre V) à la «poésie de l'expression» dans l'œuvre de Rabelais (Stapfer, 1889: chap. V); Haskovec P. (1908): "Rabelais et Jean Bouchet», Revue des études rabelaisiennes, VI, 56-60; Plattard J. (1911) : « Rabelais et Mellin de Saint-Gelais», Revue des études rabelaisiennes, IX, 90-108; Plattard J. (1912) : "Rabelais réputé poète par quelques écrivains de son temps », Revue des études rabelaisiennes, X, 291-304 ; Droz E. (1936) : «Rabelais versificateur », Humanisme et Renaissance, $\mathrm{n}^{\circ} 3,203-206$. Droz dans son article campe Rabelais en versificateur et démontrait que son art poétique s'alignait principalement sur celui de la seconde rhétorique; Guitton J. (1940) : «Le Mythe des paroles gelées », Romanic Review, 31, 1-15 ; Telle E. V. (1953) : "Thélème et le paulinisme matrimonial érasmien : le sens de l' 'énigme en prophétie'. (Gargantua, chap. LVIII) », dansFrançois Rabelais. Ouvrage publié pour le quatrième centenaire de sa mort. 1553-1953, Genève, Droz, 104-119; Screech M. (1956) : "The sense of Rabelais's Énigme en prophétie », Bibliothèque d'Humanisme et Renaissance, XVIII, 392-404 ; Mayer C. A. et Douglas C. M. (1962) : "Rabelais poète », Bibliothèque d'Humanisme et Renaissance, 23, 426-436 ; Frautschi R. L. (1963) : " The 'Enigme en prophetie' (Gargantua LVIII) and the Question of Autorship», French Studies, XVII, 331-340 ; Zeldin J. (1963) : " The Abbey and the Bottle », L'Esprit Créateur, vol. III, n², 68-74; Rawson C.J. (1965): « Rabelais and Horace. A Contact in Tiers Livre, chapter III », French Studies, 19, 373-378; Mallary Masters G. (1969): "Rabelais and the Renaissance Figure Poems », Études Rabelaisiennes, t. VIII, 51-69; Vinaver E. (1970) dans son ouvrage À la recherche d'une poétique médiévale, Paris, Nizet, 1970, 109, comparait Rabelais avec Chrétien de Troyes ; Bichon J. (1980) : «L'enracinement de Pantagruel dans les genres médiévaux », Études seiziémistes offertes à Monsieur le professeur V.-L. Saulnier par plusieurs de ses anciens doctorants, Genève, Droz, 87-89; De Rocher G. (1980) : "The Fusion of Priapus and the Muses: Rabelaisian Metaphors in the Prologue to the Quart Livre", Kentucky Romance Quarterly, $\mathrm{n}^{\circ} 4,413-420$; Demerson G. (1984) : "Tradition rhétorique et création littéraire chez Rabelais ", Etudes de Lettres, Revue de la Faculté des Lettres de l'Université de Lausanne, n`2, 3-23 ; Rigolot F. (1986) : "Énigme et Prophétie: les langages de l'hermétisme chez Rabelais », Euvres et critiques, XI, 1, 37-47; Pot O. (1988) : «Ronsard et Panurge à Ganabim », Etudes Rabelaisiennes, t. XXII, 7-26; Rigolot F. (1988) : «L'Affaire du “torchecul”': Michel-Ange et l'emblème de la charité », Études rabelaisiennes, XXI, 213-224 ; Tsuquiashi-Daddesio E. (1988) : « Le Bruissement silencieux de la graphie dans 'Les Fanfreluches antidotées", L'Esprit Créateur, vol. 28, 48-57 ; Stone D. (1991-1992) : "ThreePoets and Rabelais' Romance », Classical and Modern Literature, XII, 319325 ; Bowen B. C. (1993) : "Rabelais et le propos torcheculatif», dans Poétique et narration:
} 
reflètent à travers les «romans » rabelaisiens. L'inventaire et l'étude exhaustive de tous les poèmes qui apparaissent dans les cinq «romans » rabelaisiens restent toujours à faire. Or, cet inventaire est une condition cardinale pour énoncer de nouvelles hypothèses et perspectives quant au rôle et à la fonction de la poésie dans les " romans» de Rabelais. Deux articles, en particulier étudient en profondeur le rôle joué par la poésie chez Rabelais. Celui de Mireille Huchon est primordial, car il se propose de dégager un art poétique rabelaisien. De même, c'est la première étude per se de la poésie dans les « romans » rabelaisiens (Huchon, 1986 : 226-247 ; Droz, 1936 : 203-206). Celui d'Olivia Rosenthal, se présente tel un « début de réflexion » sur l'introduction des pièces en vers chez Rabelais (Rosenthal, $1998: 209-225)^{5}$. Il insiste également sur le lien qui s'élabore entre transmission écrite et transmission orale, à travers les poèmes des « romans » rabelaisiens.

L'écriture poétique de Rabelais au rythme syncopé, à la syntaxe parfois désarticulée, privilégie le travail sur les voix, qui tendent à brouiller les frontières entre le roman et la poésie. Elle joue sur les répétitions et les syncopes au sens musical du terme. Dans quelques poèmes, la reprise d'un ou plusieurs termes assure l'enchaînement des phrases dans le discours poétique qui confère ainsi une impression de continuité malgré sa logique heurtée. Comme l'écrit Marcel Guilbaud :

Le lecteur, en poésie, doit absolument se faire poète. Et, puisque nous ne sommes que trop instruits, c'est aux mots - qui sont maux - que nous devons nous en prendre (au besoin, par plus d'instruction, plus de mots, appeaux subtils pour prendre les mots au mot). Chassant de notre esprit et avec notre esprit les froids grêlons de la raison, essayons maintenant d'entendre les voix (Guilbaud, $1994: 24$ ).

mélanges offerts à Guy Demerson, F. Marotin et J.-P. Saint-Gérand (éds.), Paris, Champion, 371-380 ; Bowen B. C. (1998) : "Rabelais's Panurge as homo rhetoricus", dans In Laudem Caroli : Renaissance and Reformation Studies for Charles G. Nauert, J. V. Mehl (éd.), Sixteenth Century Essays \& Studies, 49, 125-133 ; Miernowski J. (1993) : «In search of a Context of Rabelaisian Hermeneutics, 'Énigme en prophétie' or How to combine the Unnameable with the Omninameable», dans Rabelais in Context, B. Bowen (sous la dir. de), Birmingham, Summer Publications, 67-77 ; Huchon M. (1996) : "La poétique dévoyée du Tiers Livre », dans Rabelais et le Tiers Livre. Actes du colloque de Nice (23 février 1996), textes réunis par E. Kotler, Paris, C.I.D, CNRS, 97-115; Duval E. (1998) : « De la Dive Bouteille à la quête du Tiers Livre », dans Rabelais pour le XXIe siècle, Actes du colloque du Centre d'Études supérieures de la Renaissance (Chinon-Tours, 1994), édités par Michel Simonin, Genève, Droz, 265-278; Pot O. (2001) : "L'Âne et le Poète : la poétique du Cinquiesme Livre », dans Le Cinquiesme Livre, Actes du colloque de Rome (16-18 octobre 1998), F. Giacone (sous la dir. de), Genève, Droz, 393-407 ; Persels J. (2002) : "Masculine rhetoric and the French 'Blason anatomique' ", dans High Anxiety. Masculinity in Crisis in Early Modern France, ( éd.) Kathleen P. Long, Kirksville, Truman State University Press, 19-35; Marrache-Gouraud M. (2003): "Hors toute intimidation ». Panurge ou la parole singulière, Genève, Droz, 45-81 et 92-116; Cornilliat F. (2011) : «Interpretation in Rabelais, interpretation of Rabelais », dans Rabelais, (éd.) J. O’Brien, Cambridge, Cambridge University Press, 43-56.

${ }^{5}$ Comme elle le précise, elle ne tient pas compte du Cinquiesme Livre. 
À bien y « écouter », le rôle de la poésie diffère légèrement au fil des « romans » de Rabelais. Dans Gargantua, Pantagruel et le Cinquiesme Livre, la poésie se manifeste surtout sous la forme d'inscriptions. Alors que dans le Tiers Livre et le Quart Livre, le caractère scripturaire de la poésie n'est plus patent. Au demeurant, il est également possible de reconnaître dans la poésie de Rabelais, tout un « folklore obscène » (Gaignebet, 1980 ; Berrong, 1986) qui, via la Friquassée Crotestyllonnée a survécu jusqu'à nos jours dans les comptines enfantines. Songeons à l' "Énigme en prophetie » du chapitre LVIII de Gargantua, laquelle, selon Thierry Martin renferme trois lectures : une partie de jeu de paume, le «maintien de la vérité divine », et une orgie achrienne (Martin, $2007: 6)^{6}$. C'est ainsi que l'écriture rabelaisienne peut se définir comme une écriture de l'impertinence et comme telle, elle subit des déviances et se retourne en « désécriture » (Glauser, 2002:12).

$\mathrm{Au}$ cœur des « romans » rabelaisiens, certains poèmes témoignent d'un ensemble cohérent qui tire son unité grâce à leur caractère poétologique. L'ombre des poètes de l'Antiquité plane sur le Prologue du Gargantua (Rabelais, Gargantua, 1994 : 7), et dans le chapitre XXVIII du Pantagruel, lequel nous est proposé la parodie des invocations adressées à l'endroit des Muses :

$\mathrm{O}$ qui pourra maintenant racompter comment se porta Pantagruel contre les troys cens geans. O ma muse, ma Calliope, ma Thalie inspire moy à ceste heure, restaure moy mes esperitz, car voicy le pont aux asnes de Logicque, voicy le trebuchet, voicy la difficulté de pouvoir exprimer l'horrible bataille que fut faicte (Rabelais, Pantagruel, $1994: 315$ ).

Le chapitre XXXI du Tiers Livre nous livre également, une allusion aux Muses :

Et me soubvient avoir leu, que Cupido quelques foys interrogé de sa mere Venus, pour quoy il n'assailloit les Muses ? respondit, qu'il les trouvoit tant belles, tant nettes, tant honestes, tant pudicques, et continuellement occupées [...] l'aultre à invention Rhetoricque, l'aultre à composition Poëticque, l'aultre à disposition de Musique : que approchant d'elles, il desbandoit son arc, fermoit sa trousse, et extaignoit son flambeau par honte et craincte de leurs nuire (Rabelais, Tiers Livre, $1994: 451)$.

${ }^{6}$ Pour d'autres lectures possibles, voir les études de Screech M. A. (1956): "The sense of Rabelais's Énigme en prophétie », Bibliothèque d'Humanisme et Renaissance, XVIII, 392-404; Rigolot F. (1986) : " Énigme et Prophétie: les langages de l'hermétisme chez Rabelais », Euvres et critiques XI1, 37-47 ; Miernowski J. (1993) : «In search of a Context for Rabelaisian Hermeneutics: 'Énigme en prophétie' or How to Combine the Unnameable with the Omninameable», dans Rabelais in Context. Proceedings of the 1991 Vanderbilt Conference, éd. Bowen B. C., Alabama: Summa Publications, Birmingham, 67-77 ; Pot O. (2008) : "'L'énigme en prophétie' chez Rabelais: naissance et destin d'un genre ", dans L'énigmatique à la Renaissance : formes, significations, esthétique, éd. Martin D., Servet P., Tournon A., Champion, Paris, 137-154 ; Cornilliat F. (2011) : «Interpretation in Rabelais, interpretation of Rabelais », dans The Cambridge Companion to Rabelais, éd. O'Brien J., Cambridge University Press, Cambridge, 43-48. 
Quant au Quart Livre, dans les chapitres finals (Rabelais, Quart Livre, 1994 : 695-701), la poésie est reine, ainsi, l'île de Ganabin, ultime île évoquée alors que « continuant le bon vent, et ces joyeulx propous », Pantagruel prend soin d'établir un parallèle avec le séjour des Muses : " Voyez vous cy davant à Orche ce hault rochier à deux crouppes bien ressemblant au mons Parnasse en Phocide ? ». Et Xenomanes de préciser: "Y est toutesfoys vers ceste crouppe dextre la plus belle fontaine du monde ». L'œuvre poétique de Rabelais reflète un double mode d'insertion et d'inscription. Dans le Pantagruel et le Gargantua, le caractère scripturaire et allégorique des poèmes est patent.En effet, ceux-ci se présentent constamment sous la forme d'inscriptions (Hervé, 2012 : 1-12).

L'un des principaux emblèmes caractéristiques du début du Gargantua est le chapitre II (Rabelais, Gargantua, 1994 : 11-14). Ce poème est un plaquage monté et inséré qui renverse les notions traditionnelles de narration. Dans les éditions de 1535 et 1537, il est imprimé de façon différente du texte de Gargantua, ce qui traduit son côté énigmatique et insolite. Rabelais semble accorder à ce poème une importance capitale à cause de sa place dans l'œuvre. Le chapitre est placé visuellement et graphiquement au cœur des problèmes de l'écriture. Jean Audeau, qui fut peut-être un des voisins de Rabelais (Bon, 2006 : 292-302), trouve « en un pré qu'il avoit prés l'arceau gualeau [...] un grand tombeau de bronze long sans mesure », contenant «neuf flaccons ». Celui du milieu « couvroit un gros, gras, grand, gris, joly, petit, moisy livret» (10), dans lequel se dissimule la « genealogie » de Gargantua, et à «la fin du livre estoit un petit traicté intitulé, Les Fanfreluches antidotées », dont les « ratz et blattes ou [...] aultres malignes bestes avoient brousté le commencement » (11). «Les Fanfreluches antidotées » se rattachent d'une certaine façon à la lignée de la fatrasie et du coq-à-l'âne et même de la sotte chanson. Ce poème met en relief le soin de l'auteur à exploiter toutes les formes possibles visuelles, graphiques et phoniques de détourner l'écriture. Ce poème devient une sorte de texte dans le texte. L'ordre métrique, la simplicité et les effets d'écho sonore des mots vont de pair avec une émotion authentique qui ne se laisse guère éteindre ou plutôt dompter par la construction poétique. Le poète est celui qui, à force de méticulosité et de rigueur dans son travail lexical et stylistique, parvient au miracle de la formulation, miracle au sens de merveille gracieuse, épurée de toute contingence. Rabelais est bel et bien ce poète-artiste et artisan car il se donne pour matériau de travail un substrat verbal.

Que l'on songe également au titre du chapitre LIIII (Rabelais, Gargantua, 1994 : 141-144) : «Inscription mise sus la grande porte de Theleme ». Destinée au linteau de la porte de Thélème, la forme des vers doit convoquer et provoquer l'œil du lecteur. Celle-ci nous est présentée comme écrite " en grosses lettres antiques ». C'est un emblème qui promeut Thélème. Les quatorze strophes de ce chapitre sont composées de rimes équivoquées ainsi que de rimes batelées. Cette pièce en vers figure parmi les plus longues de toute l'œuvre de Rabelais et occupe pour ainsi dire tout le chapitre LIIII. L'Abbaye de Thélème a toujours su attiser la faveur des esprits libres. Ainsi, des écrivains comme Charles Vildrac, Georges Duhamel, René Arcos, Henri-Martin Barzun et Alexandre Mercereau, mais aussi un peintre Albert 
Gleizes, un musicien Albert Doyen et un typographe Lucien Linard fondèrent en 1906 un domaine près de Créteil et entreprirent d'y vivre en phalanstère. Ils la nommèrent l'Abbaye en souvenir de Rabelais et de Thélème. Ils eurent également pour premier soin de clouer sur la porte de leur phalanstère un écriteau, sur lequel était reproduit plusieurs vers de l'Inscription mise sus la grande porte de Theleme (Sénéchal, 1930).

L'inscription, tout comme l'épisode de l'abbaye de Thélème, constitue le poème le mieux étudié de l'œuvre de Rabelais. L'épisode de l'inscription est considéré comme un morceau de choix par les critiques, en tout particulier pour ceux qui considèrent Rabelais comme « le dernier des Grands Rhétoriqueurs». Jean Molinet, Jean Lemaire de Belges et Octovien de Saint-Gelais, parmi tant d'autres, semblent avoir été ses modèles poétiques. En effet, on n'a pas fini de dénombrer les traits qui caractérisent en ce poème l'esthétique du Grand Rhétoriqueur. «Utopie » (Berrong, 1985: 22), « rêve » (Glauser, 1966: 36), « poème » (Morçay, 1949: XXXV), « farce» (Desonay, 1953: 102), Thélème a même été qualifié de «joli et poétique joujou» (Thuasne, $1970: 370$ ). Diane Desrosiers-Bonin, pour sa part, l'assimile à une forme littéraire, le «Temple », sur le modèle des genres que pratiquaient les Grands Rhétoriqueurs (Desrosiers-Bonin, 1998 : 242).

Le caractère scripturaire de la poésie est encore plus manifeste dans le Pantagruel. De l'épitaphe de Badebec vouée à « estre engravé » et qui a l'apparence d'un éloge funèbre, comme l'atteste la présence du registre épidictique repérable dans diverses expressions à connotations valorisantes, mais il s'agit en fait d'un poème purement comique car les divers éléments de cette prosopographie donnent d'elle une image hétéroclite et peu avenante, conforme à la tonalité burlesque de ce poème (Rabelais, Pantagruel, 1994 : 226), en passant par le rondeau double ou rondeau cinquain de décasyllabes que Panurge offrit à la dame Parisianne " par escript en la forme que s'ensuyt » (Rabelais, Pantagruel, 1994 : 295-297), sans oublier les deux trophées ou «dicton victorial » du chapitre XXVII (Rabelais, Pantagruel, 1994 : 308-311), l'un étant composé par Pantagruel, l'autre par Panurge, le second étant la parodie du premier. Les deux trophées sont pourvus de la même structure, ainsi, ils contiennent tout d'abord un huitain de décasyllabes, puis un sizain de tétrasyllabes et enfin, un quatrain d'octosyllabes. En réalité, le terme « trophée » désigne à la fois l'inscription qui orne le monument et la construction en tant que telle. Il renvoie à la fois à une longue tradition poétique d'origine hellénique réactivée au Moyen Âge puis à la Renaissance par les rhétoriqueurs, et à une pratique sociale antique, véritable anthropologie de la victoire. Rabelais incorpore aussi dans la trame romanesque un «blason et divise» que Pantagruel compose pour les «licentiez» de l'Université d'Orléans (Rabelais, Pantagruel, 1994 : 232), de même pour le quatrain du chapitre XXX (Rabelais, Pantagruel, 1994: 325), pour la devise d'Épictète : «Au dessus de la treille estoient pour sa devise ces vers escriptz ». Ainsi, les poèmes apparaissent d'abord comme transmis par écrit, alors que dans le Tiers Livre et le Quart Livre, les poèmes circulent par voie orale. Au demeurant, dans le Tiers Livre et le Quart Livre, les poèmes sont bien plus nombreux et variés. 
Le Tiers Livre s'ouvre sur un dizain de « François Rabelais à l'esprit de la royne de Navarre ». Il se termine sur une pièce poétique. Le huitain final, en décasyllabes, à la gloire du pantagruélion, inspiré de l'éloge virgilien appartient, tout comme le dizain liminaire, à la rhétorique encomiastique (Rabelais, Tiers Livre, 1994 : 512). Le huitain en décasyllabes au chapitre VIII (Rabelais, Tiers Livre, 1994 : 376) est un emprunt à un ouvrage collectif de 1543, la Fleur de poesiefrançoyse. Recueil joyeulx contenant plusieurs Huictains, Dixains, Quatrains, Chansons et autres dictéz de diverses matieres mis en nottesmusicalles par plusieurs autheurs. Les vers de la Sibylle sont également sous forme de huitain, avec alternance de vers de quatre et de trois syllabes. L'hétérométrie concourt à faire de ces vers une parole oraculaire. Puis, sous forme de versets Panurge interprète ensuite « les dictz propheticques » (Rabelais, Tiers Livre, 1994 : 404). Les quatrains, comme les huitains se caractérisent par leur variété. Celui du chapitre VI (Rabelais, Tiers Livre, 1994 : 371), en octosyllabes est donné comme provenant de la marraine du grand-père de Panurge. Le métadiscours poétique est également présent. La pratique poétique du Tiers Livre par exemple, réside pour l'essentiel, dans l'imitation, comme en témoigne l'introduction de traductions versifiées de vers grecs et latins : de Virgile, en passant par Sénèque, sans oublier Martial, mais surtout d'Homère et Virgile (Rabelais, Tiers Livre, 1994 : 379-382 et 384-388). Qui plus est, l'union de la musique et de la poésie parcourt également ces deux « romans ». Du « dizain jolliet» (Rabelais, Tiers Livre, 1994: 640), composé par Panurge sur l'île de Ruach («vent» ou « esprit» en hébreu), île sur laquelle, il nous sera livré une définition du sonnet (Rabelais, Tiers Livre, 1994 : 639), en passant par « un Epode » chanté « melodieusement » à la louange des « sacrosainctes Decretales » (Rabelais, Tiers Livre, 1994 : 657), sans oublier les « Dithyrambes, Craepalocomes, Epaenons » chantés « soubs la conduicte de Manduce » (Rabelais, Tiers Livre, $1994: 676$ ).

Quant au Cinquiesme Livre (1564) celui-ci est doté d'une coloration poétique vivace puisque la production poétique se déploie avec plus d'éclat que jamais à partir du chapitre XLIIII. Elle acquiert un rôle essentiel dans le dénouement de la quête de Panurge. La poétique de l'inscription joue un rôle prépondérant. Ainsi, le chapitre XXXIII contient deux vers inscrits « en la face de l'arc dessous le zoophore " (Rabelais, Cinquiesme Livre, 1994 : 809), par ailleurs, le blason de Chinon est formé par un quatrain (Rabelais, Cinquiesme Livre, 1994 : 810). Dans une « des tables susdites à dextre » du temple, c'est un "vers Iambique senaire » qui est « insculpé en lettres Latines antiquaires » (Rabelais, Cinquiesme Livre, 1994 : 815). Ce vers nous est donné en latin et il est accompagné d'une traduction en français. Des genres qui appartiennent à l'Antiquité sont évoqués au chapitre XXXIX : « en joyeux Epinicies, et petites chansons villatiques et dithyrambes resonnans » (Rabelais, Cinquiesme Livre, 1994: 821). Certaines pièces sont des imitations ou des emprunts. Il nous suffit de songer par exemple, au septain incomplet du Prologue présenté comme un emprunt à la Cornemuse des Prelats (Rabelais, Cinquiesme Livre, 1994 : 724). Le chapitre VI (Rabelais, Cinquiesme Livre, 1994 : 738-739) se clôt sur une épigramme de Victor Brodeau. La fin du Cinquiesme Livre se caractérise surtout par la présence des mystères bachiques. 
Notons que, dans l'œuvre rabelaisienne, trois pièces qui ne sont pas stricto sensu versifiées, mais qui entrent de toute évidence en résonance avec des "genres " poétiques de l'époque, sont présents : il s'agit des blasons (Demonet, 1988 : 87-94; Roudaut, 1996: 3-9; Marrache-Gouraud, 2000: 153-164) ${ }^{7} \mathrm{du}$ Tiers Livre et de l'anatomie de Quaresmeprenant dans le Quart Livre (Rabelais, Quart Livre, 1994 : 608-615).Dans le Tiers Livre, on peut trouver deux types de blasons : le premier est illustré par les deux blasons du couillon (blason de Panurge au chapitre XXVI, Rabelais, Tiers Livre, 1994 : 432-435, et le blason de Frère Jean au chapitre XXVIII, Rabelais, Tiers Livre, 1994 : 438-443) ces deux blasons portent sur des parties du corps ; le second est caractérisé par le blason du fou à deux voix du chapitre XXXVIII (Rabelais, Tiers Livre, 1994 : 470-474).

Les « romans » rabelaisiens méritent d'être traités certes, comme œuvre littéraire, mais aussi et surtout comme production en prose rimée faite pour être entendue. C'est ainsi que la poésie, à travers les « romans » rabelaisiens sort grandie, ou plutôt elle ressort agrandie, car elle est capable d'accueillir en son sein les alliances de tous les genres. Somme toute, à travers les « romans » de Rabelais, qu'il s'agisse de longues énumérations, de fatras, de baragouins, de poèmes, de farce, de mystère, du mélange vers-prose, tous ces modes d'énonciation traduisent une espèce de dédoublement du langage. Comme le note Pascale Mounier, Rabelais se plaît, dans ses « romans » à instaurer :

une rivalité entre sa production et des formes prestigieuses de composition et brouille les pistes de catégorisation. Quel genre crée-t-il? Difficile à dire. Une série, en tout cas, comme l'attestent les appellations Tiers, Quart, et Cinquiesme livre. Ses textes, publiés entre 1532 et 1564, ont en outre différents points communs : la fiction, la narration, l'écriture en prose et l'assignation d'une portée idéologique aux aventures. Peut-on aller plus loin que ces remarques d'ordre général? (Mounier, $2008: 773)$.

La prise en considération de l'ensemble des pratiques d'écriture rabelaisiennes permet de conférer une nouvelle configuration, une nouvelle poétique et de nouvelles propriétés à l'œuvre rabelaisienne. Mieux encore, entre " vers carminiformes » (Rabelais, Gargantua, $1994: 53$ ) et « oraison soluë» (Rabelais, Cinquiesme Livre, 1994 : 726), entre poésie et prose, l'œuvre rabelaisienne gagne à être lue et être caractérisée comme une tentative pour élaborer une complétude ou plutôt une plénitude, toujours atteinte : la proésie. Dès lors :

\footnotetext{
${ }^{7}$ Voir les analyses de Marie-Luce Demonet, "Le 'Blason du Fou' (Tiers Livre, ch.38), binarité et dialogisme", dans L'Intelligence du passé, les faits, l'écriture et le sens. Mélanges offerts à Jean Lafond par ses amis (Tours: Presses de l'Université de Tours, 1988), pp. 87-94 ; François Roudaut, "Quelques remarques sur le 'blason du fou'. Rabelais, Le Tiers Livre, chapitre XXXVIII", dans Information littéraire, XLVIII-2 (mars-avril 1996) pp. 3-9; Myriam Marrache-Gouraud, "Trois détours signifiants: les blasons de Rabelais”, dans Le détour, La Licorne, 54 (2000), pp. 153-164.
} 
Le regard du poète est décapé des traces de routine, prêt à l'étonnement, à tout émerveillement. La poésie excède le langage poétique qui, à la manière de chaque démarche artistique, n'en est qu'une incarnation provisoire. [...] La lecture ou l'audition d'un " vrai » poème nous restitue en un éclair la vigueur de cette réalité et nous buvons les mots à même la source, nous éprouvons une infinie reconnaissance à l'égard de celui qui a rendu souplesse, saveur et vie " courante » à ce qui était figé sous la croûte des conventions (Nys-Mazure, $2004: 213-214$ ).

Il y a en effet, dans la poésie rabelaisienne une recherche constante du plaisir de la surprise : le poème est en quelque sorte la promesse d'une jouissance toujours inaccomplie voire inavouée et donc toujours possible. La poésie dans les « romans » rabelaisiens qui est avant tout création, se donne pour ambition de susciter d'autres mondes, et d'autres modes d'énonciation. Carrefour des cultures, carrefour des traditions, carrefour des " genres », carrefour des techniques, l'œuvre de Rabelais mérite à tous égards le titre d'œuvre-carrefour. Somme toute, la question du rapport de la prose à la poésie dépasse chez Rabelais, les catégories habituelles de la rhétorique et de la poétique. Chez Rabelais, la poésie ne serait-elle pas carrefour de sens et de voix, vouée à dire la promesse d'une rencontre ? Dès lors, cette œuvrecarrefour, n'annoncerait-elle pas un nouveau «genre » littéraire encore à venir ?

\section{RÉFÉRENCES BIBLIOGRAPHIQUES}

Berrong, R. M., (1985) Every Man for Himself: Social Order and Its Dissolution in Rabelais. Stanford, Stanford French and Italian Studies 38.

Berrong, R. M., (1986) Rabelais and Bakhtin: Popular Culture in Gargantua and Pantagruel. Lincoln/London, University of Nebraska Press.

Bon, F. (2006) «139. Souvenirs de Jean Audeau un spécialiste ignoré de Rabelais » in Bon, F., Tumulte. Paris, Fayard, pp. 292-302.

Chambon, J.-P., (1993) «Andrieu de la Vigne, source de Rabelais ? » in Revue des études rabelaisiennes. T. XXIX, pp. 57-61.

Cornilliat, F., (1994) "Or ne mens ». Couleur de l'Éloge et du Blâme chez les " Grands Rhétoriqueurs ». Paris, Champion.

Cornilliat, F., (2002) « Au-delà de la rhétorique ? La poésie de la Renaissance et le syndrome de Monsieur Jourdain » in Rhetorica. 20-4, pp. 335-356.

Cornilliat, F., (2011) "Interpretation in Rabelais, interpretation of Rabelais" in O'Brien, J. (ed.), The Cambridge Companion to Rabelais. Cambridge, Cambridge University Press, pp. 43-48.

Cornulier, B. de, (2000) «Rabelais Grand Rhétoriqueur. L'enchaînement dans l'Inscription mise sur la grande porte de Thélème ». Études Rabelaisiennes. T. XXXIX, pp. 111-124.

Demonet, M.-L., (1988) «Le 'Blason du Fou' (Tiers Livre, ch.38), binarité et dialogisme " in Aquilon, P. et al. (éd.), L'Intelligence du passé, les faits, l'écriture et le sens. Mélanges offerts à Jean Lafond par ses amis. Tours, Presses de l’Université de Tours, pp. 87-94. 
Desonay, F., (1953) «En relisant L'Abbaye de Thélème... » in François Rabelais. Ouvrage publié pour le quatrième centenaire de sa mort. 1553-1953. Genève, Droz.

Desrosiers-Bonin, D., (1998) «L'Abbaye de Thélème et le Temple des Rhétoriqueurs "), dans Rabelais pour le XXIe siècle, Actes du colloque du CESR (1994), éd. Michel Simonin, Études rabelaisiennes, t. XXXIII, Droz, Genève, 241-248.

Gaignebet, C., (1980) Le folklore obscène des enfants. $1^{\text {ère }}$ édition 1974. Paris, Maisonneuve et Larose.

Glauser, A., (1966) Rabelais créateur. Paris, Nizet.

Glauser, A., (2002) Écriture et désécriture du texte poétique de Maurice Scève à Saint John Perse. Paris, Nizet.

Guilbaud, M., (1989) Le Clown Françoys ou Rabelais et le sens agile, tome I : La fête foraine.Paris, Guilbaud.

Guilbaud, M., (1994) Rabelais sur la lyre d'Orphée. Paris, Guilbaud, Paris.

Haskovec, P., (1908) «Rabelais et Jean Bouchet» in Revue des études rabelaisiennes. VI, pp. 56-60.

Hervé, N., (2012) «Insertions et inscriptions : une étude métrique des poèmes du Gargantua » in Le Verger [En ligne]. $\mathrm{N}^{\circ} 1$, pp. 1-12, disponible sur: http://www.cornucopia16.com/a-le-verger-revue-en-ligne/le-verger-bouquets/jan v-2012-nathalie-herv\%C3\%A9/ [Dernier accès le 6 janvier 2012].

Huchon, M., (1986) «Rabelais et les 'genres d'escrire' »in La Charité, R. C. (éd.), Rabelais's Incomparable Book. Lexington, French Forum Publishers, pp. 226247.

Jeanneret, M., (1987) Des mets et des mots. Banquets et propos de table à la Renaissance. Paris, Corti.

Lebègue, R., (1958) «Rabelais et les grands rhétoriqueurs » in Lettres romanes. $\mathrm{N}^{\circ} 12$, pp. $5-18$.

Lecointe, J., (2001) «La poésie parmi les arts au XVIe siècle » in Galand-Hallyn, P. et F. Hallyn (éd.), Poétiques de la Renaissance: le modèle italien, le monde franco-bourguignon et leur héritage en France au XVI siècle. Genève, Droz, pp. 53-71.

Marrache-Gouraud, M., (2000) « Trois détours signifiants: les blasons de Rabelais » in La Licorne. $\mathrm{N}^{\circ} 54$, pp. 153-164.

Martin, T., (2007) Poésie homosexuelle en jobelin : de Charles d'Orléans à Rabelais. Montpellier, GKC/Question de Genre.

Molino, J., (1993) « Les genres littéraires » in Poétique. Nº3, pp. 3-28.

Mounier, P., (2008) «L'Héroïsme guerrier dans les cinq Livres: la fiction rabelaisienne au défi des genres » in Revue d'histoire littéraire de la France. $\mathrm{N}^{\circ} 4$, vol. 108, pp. 771-788.

Nys-Mazure, C., (2004) La chair du poème. Petite initiation à la vie poétique. Paris, Albin Michel.

Pilar Suárez, M., (2006) «Les Enjeux du décepteur : Panurge, Gargantua, Francion » in Thèlème. Revista Complutense de Estudios Franceses. N²1, pp. 199212. 
Plattard, J., (1911) «Rabelais et Mellin de Saint-Gelais » in Revue des études rabelaisiennes. T. IX, pp. 90-108.

Plattard, J., (1912) «Rabelais réputé poète par quelques écrivains de son temps » in Revue des études rabelaisiennes. T. X, pp. 291-304.

Rabelais F., (1949) L'Abbaye de Thélème, $2^{\mathrm{e}}$ édition revue et augmentée, publiée par R. Morçay. Genève, Droz.

Rabelais F., (1994) CEuvres complètes, M. Huchon (éd.) et F. Moreau (collab.). Paris, Gallimard, Coll. Bibliothèque de la Pléiade.

Rigolot, F., (1982) Le Texte de la Renaissance. Des Rhétoriqueurs à Montaigne. Genève, Droz.

Rosenthal, O., (1998) « Rabelais poète ou les modes d'insertion de la poésie dans l'œuvre de Rabelais » in La Licorne. N 46, pp. 209-225.

Rothstein, M., (1996) «Le genre du roman à la Renaissance» in Le Roman chevaleresque tardif, Études françaises. $\mathrm{N}^{\circ} 1$, vol.32, pp. 35-47.

Roudaut, F., (1996) «Quelques remarques sur le 'blason du fou'. Rabelais, Le Tiers Livre, chapitre XXXVIII » in Information littéraire. XLVIII-2, pp. 3-9.

Sénéchal, C., (1930) L'Abbaye de Créteil. Paris, André Delpeuch.

Slerca, A., (1996) "Guillaume Cretin e François Rabelais» in Studi francesi. $\mathrm{N}^{\mathrm{o}} 120$, pp. 586-589.

Thuasne, L., (1911) Villon et Rabelais. Paris, Fischbacher.

Thuasne, L., (1970) Études sur Rabelais. Paris, Champion. 